\title{
SMF/MMF Based In-building Gigabit Wireless Access Systems Using Simplified 60-
} GHz Transceivers

\author{
Pham, Tien Thang; Lebedev, Alexander; Beltrán, M.; Yu, Xianbin; Llorente, R.; Tafur Monroy, Idelfonso
}

Published in:

Proceedings of the European Conference on Optical Communication (ECOC) 2011

Publication date:

2011

Document Version

Publisher's PDF, also known as Version of record

Link back to DTU Orbit

Citation (APA):

Pham, T. T., Lebedev, A., Beltrán, M., Yu, X., Llorente, R., \& Tafur Monroy, I. (2011). SMF/MMF Based Inbuilding Gigabit Wireless Access Systems Using Simplified 60-GHz Transceivers. In Proceedings of the European Conference on Optical Communication (ECOC) 2011 (pp. We.10.P1.111). Optical Society of America. http://www.ecoc2011.org/

\section{General rights}

Copyright and moral rights for the publications made accessible in the public portal are retained by the authors and/or other copyright owners and it is a condition of accessing publications that users recognise and abide by the legal requirements associated with these rights.

- Users may download and print one copy of any publication from the public portal for the purpose of private study or research.

- You may not further distribute the material or use it for any profit-making activity or commercial gain

- You may freely distribute the URL identifying the publication in the public portal 


\title{
SMF/MMF Based In-building Gigabit Wireless Access Systems Using Simplified 60-GHz Transceivers
}

\author{
T.T. Pham ${ }^{1}$, A. Lebedev ${ }^{1}$, M. Beltrán ${ }^{2}$, X. Yu ${ }^{1}$, R. Llorente ${ }^{2}$ and I. Tafur Monroy ${ }^{1}$ \\ ${ }^{1}$ DTU Fotonik-Department of Photonics Engineering, Technical University of Denmark, DK-2800, Kgs. Lyngby, Denmark. \\ E-mail:ptit@fotonik.dtu.dk \\ ${ }^{2}$ Valencia Nanophotonics Technology Center, Universidad Politécnica de Valencia, Camino de Vera s/n, 46022 Valencia, Spain. \\ E-mail: mbeltran@ntc.upv.es
}

\begin{abstract}
Hybrid fiber-wireless transmission of 2-Gbps signals in 60-GHz band over a composite channel (10-km SMF/1-km MMF/6.5-m wireless) is experimentally demonstrated for gigabit inbuilding wireless access using simple direct modulation, optical upconversion and envelope detection.

OCIS codes: (060.2330) Fiber optics communications; (060.5625) Radio frequency photonics.
\end{abstract}

\section{Introduction}

Millimeter-wave (MMW) wireless communication is receiving tremendous research interests to explore the potential of broadband wireless data transmission in future wireless personal area networks (WPAN) to support bandwidth-hungry services such as high-definition television (HDTV), 3D gaming and high-speed data access. Due to the high atmospheric loss of MMW signal, radio-over-fiber (RoF) technique is generally considered as an attractive solution to extend the reach of MMW wireless networks. Several MMW RoF systems have been proposed and demonstrated recently in the literature [1-4]. Additionally, in all MMW bands, 60-GHz band has been considered to be standardized for future WPAN networks by several working groups such as ECMA, WirelessHD and IEEE 802.15.3c [5-7]. In order to commercialize 60-GHz RoF systems for next-generation high-speed wireless networks, it is highly desirable to develop a system with less complexity and low power consumption transceivers as a large number of remote antenna units (RAUs) and user terminals are to be deployed in the networks.

Regarding the optical fiber plant, single-mode fibers (SMFs) have been widely deployed in optical core/metro/access networks, and well studied in the previously proposed MMW RoF systems. On the other hand, multi-mode fibers (MMFs) are predominantly deployed in in-building networks as a backbone for local area networks (LANs) (approximately from $85 \%$ to $90 \%$ ) due to several advantages such as low cost, easy installation and maintenance [8]. When developing a system to meet the increasing demand for high-bandwidth services, it is greatly encouraged to efficiently utilize this existing infrastructure. Together with SMFs in access networks, the reuse of MMF for distribution of MMW signals through in-building optical networks to WPAN networks will tremendously save fiber reinstallation cost. The schematic of such system is presented in Fig. 1. MMW signal is generated optically at the central office (CO) and then distributed to user terminals through a composite channel including SMF, MMF and wireless link. Several experiments on MMF based RoF systems have been reported [9-11 and references therein]. However, to the best of our knowledge, $60-\mathrm{GHz}$ over MMF system has not been demonstrated yet. The reported highest frequency and data rate so far are $38.8 \mathrm{GHz}$ and $900 \mathrm{Mbps}$ over 100-m MMF [10]. Data transmission over a composite channel has been demonstrated at low bitrate and low RF frequency: 120Mbps data at $31.14 \mathrm{GHz}$ over 20-km SMF plus 300-m MMF and 3-m wireless link [11]. For future deployment of $60-\mathrm{GHz}$ system, it is important to evaluate the performance of $60-\mathrm{GHz}$ signal over such composite channel.

In this paper, for the first time, we report transmission of gigabit data at $60 \mathrm{GHz}$ band with simplified transceivers in end-to-end composite RoF network scenario: access and in-building networks using different types of optical fibers. The link consists of 10-km SMF, 1-km MMF and 6.5-m wireless. Direct modulation and double-sideband suppressed carrier (DSB-SC) scheme are adopted in the central office (CO) to optically generate $60-\mathrm{GHz}$ subcarrier while simple envelope detection is employed at user terminals. We show that error-free transmission of 2-Gbps data was successfully achieved over the link with less than $4-\mathrm{dB}$ power penalty due to accumulated fiber dispersion.

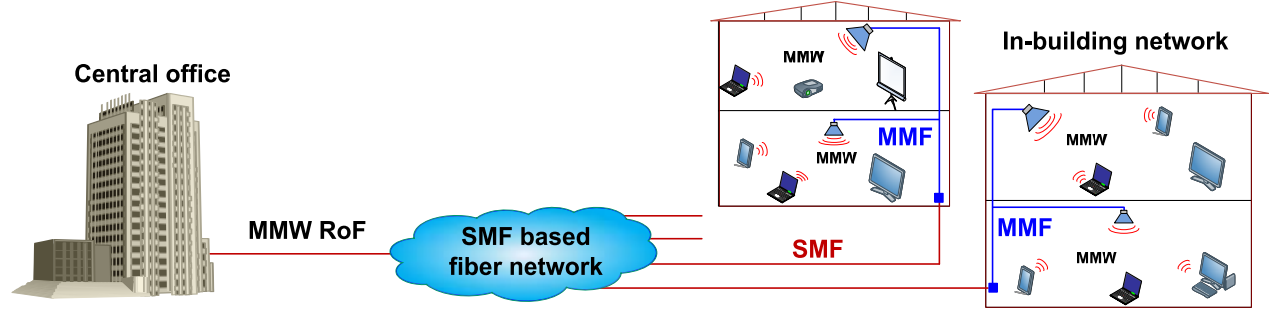

Fig. 1. Distribution of MMW RoF signals over combined SMF and MMF link for in-building broadband wireless access. 


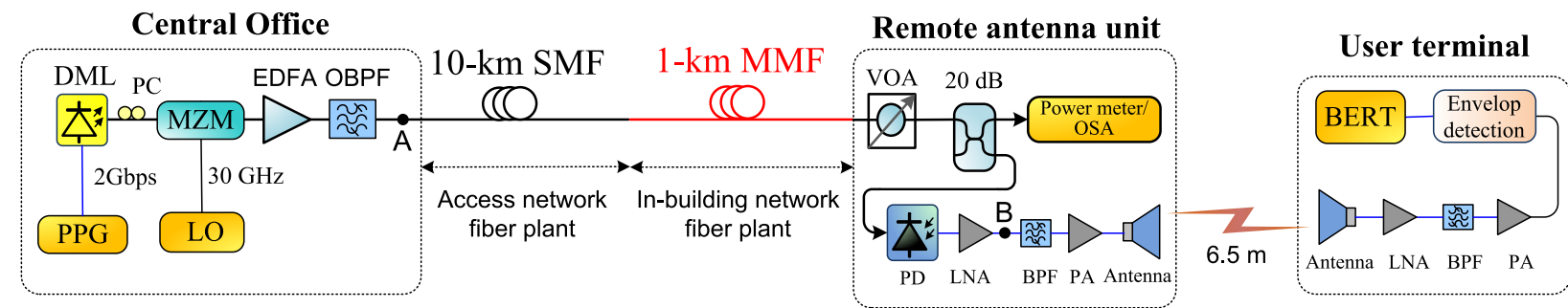

Fig. 2. Experimental setup: PPG: pulse pattern generator, DML: directly-modulated laser, PC: polarization controller, MZM: Mach-Zehnder modulator, EDFA: Erbium doped fiber amplifier, OBPF: optical band-pass filter, VOA: variable optical attenuator, OSA: optical spectrum analyzer, PD: photo-detector, LNA: low-noise amplifier, PA: power amplifier, BPF: band-pass filter, BERT: bit-error-rate tester.

\section{Experimental setup}

Fig. 2 illustrates the experimental setup. At the CO, 2-Gbps PRBS baseband data from a pulse pattern generator (PPG) with a word length of $2^{31}-1$ directly modulated a $1.55-\mu \mathrm{m}$ laser. To generate a $60-\mathrm{GHz}$ subcarrier, the wellknown DSB-SC scheme was utilized. The output from the laser was launched to a Mach-Zehnder modulator (MZM) which was biased at the minimum transmission point and driven by $30-\mathrm{GHz}$ sinusoid signal from a local oscillator (LO). The output of the MZM was amplified and filtered out amplified spontaneous emission (ASE) noise by an optical band-pass filter (OBPF) (0.8-nm bandwidth). The optical signal was then distributed to a RAU over an optical fiber link composed of 10-km standard SMF and additional 100-m or 1-km MMF. The diameters of core and cladding of the MMF are 50 and $125 \mu \mathrm{m}$, respectively. At the RAU, the optical signal was detected by a broadband $75-\mathrm{GHz}$ photo-detector (PD). The detected $60-\mathrm{GHz}$ signal was amplified and radiated to the air by a horn antenna and collected by another one. The wireless link was $6.5 \mathrm{~m}$, which was limited by the available lab space. The received wireless signal was then again amplified and filtered. To simplify the end-user wireless terminal, electrical envelope detection was employed to down-convert $60-\mathrm{GHz}$ band signal to the baseband. The output signal of the envelope detector was fed to a bit-error-rate tester (BERT) to evaluate the system performance. Both the antennas have $20-\mathrm{dBi}$ gains in $50-75 \mathrm{GHz}$ frequency range and $12^{\circ}$ beam width. All the amplifiers have 10-GHz bandwidth $(55-65 \mathrm{GHz})$. The power amplifiers (PA) have 28.7-dB gain and the low noise amplifiers (LNA) have 16-dB gain. Bandwidth of the BPFs is $5.7 \mathrm{GHz}(56.3-62 \mathrm{GHz})$.

\section{Experimental results and discussion}

Fig. 3(a) shows the optical spectra at the output of the MZM with and without the 30-GHz LO signal. When the LO signal was applied, two first-order sidebands were dominant and the original optical carrier at $1549.75 \mathrm{~nm}$ was suppressed more than $13 \mathrm{~dB}$.

In general, it is important to generate high quality RF subcarrier to achieve high performance data transmission. The phase noise from the LO does not change the amplitude of a RF signal. However, fiber dispersion such as chromatic dispersion of the SMF, modal dispersion of the MMF causes the phase-to-intensity conversion that, in turn, makes the amplitude of the RF signal vary after fiber transmission. Fig. 3(b) shows the measured phase noise of the $30-\mathrm{GHz}$ subcarrier from the $\mathrm{LO}$ as well as the generated $60-\mathrm{GHz}$ subcarriers at back-to-back (B2B) and after fiber transmission. It is obvious that the quality of the $60-\mathrm{GHz}$ subcarrier was maintained after fiber transmission. For example, at $100-\mathrm{kHz}$ offset, the phase noise of the $60-\mathrm{GHz}$ subcarrier was only about $5 \mathrm{~dB}$ higher than that of the $30-\mathrm{GHz}$ LO. Therefore, we can use the $30-\mathrm{GHz}$ subcarrier from a LO which has similar phase-noise characteristic as the requirement for the $60-\mathrm{GHz}$ subcarrier.

The electrical spectra of the signals at point B in Fig. 2 in cases of B2B and fiber transmission at -3-dBm optical power are shown in Fig. 3(c). The RF spectrum was maintained after 10-km SMF and 100-m MMF. However, there was a slight distortion in the case of 10-km SMF and 1-km MMF due to large modal dispersion of the MMF.

Fig. 4 shows the measured BER curves and eye-diagrams of 2-Gbps data in different transmission cases: B2B, SMF only, MMF only and combined SMF/MMF. The optical power to the PD was controlled by a variable optical attenuator (VOA). We can observe that chromatic dispersion of 10-km SMF introduced less than 1-dB power penalty. Additional 100-m MMF caused about $0.5-\mathrm{dB}$ power penalty. Suffered by large modal dispersion, the BER performance after 1-km MMF transmission had about 2-dB power penalty. In the case of combined 10-km SMF and 1-km MMF transmission which has both large chromatic dispersion and modal dispersion, the BER curve shows a slightly different slope and the power penalty at a BER of $10^{-9}$ were approximately $4.3 \mathrm{~dB}$. Additionally, we observed in the experiment that the BER performance rapidly became worse when the received optical power was higher than about $-3.0 \mathrm{dBm}$. It is because the transmitted power of the $60-\mathrm{GHz}$ signal was saturated in the current RF configuration when high optical power was launched to the PD.

Apart from the utilization of both SMF and MMF plants, this system architecture has some additional important features. Firstly, the use of direct modulation for data and optical frequency up-conversion does not require high 
speed laser. Secondly, the DSB-SC scheme enables the use of low RF frequency LO and avoiding chromatic dispersion induced RF power fading [3-5]. In a wavelength-division multiplexing (WDM) system scenario, the MZM can be shared by several channels. Thirdly, envelope detection is a simple demodulation method, which is transparent to RF frequency and does not require any phase-locked LO and mixer for down-conversion. Therefore, in connection with high transmission performance, this scheme is expected to be a very cost-effective solution for broadband $60-\mathrm{GHz}$ in-building wireless networks with the use of simple transceiver, as well as long access reach.

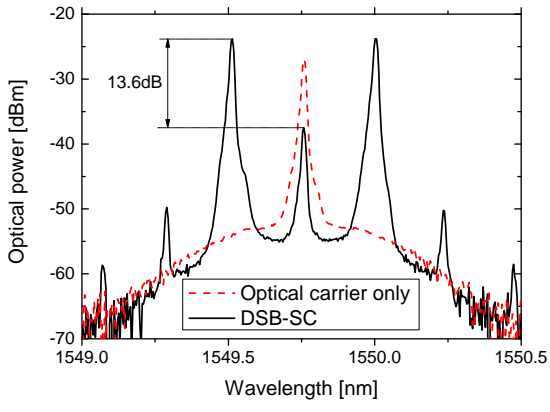

a)

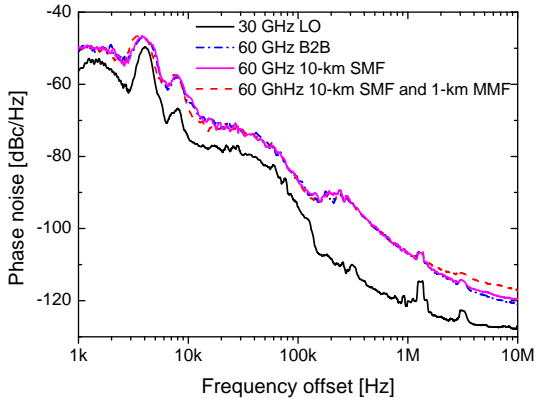

b)

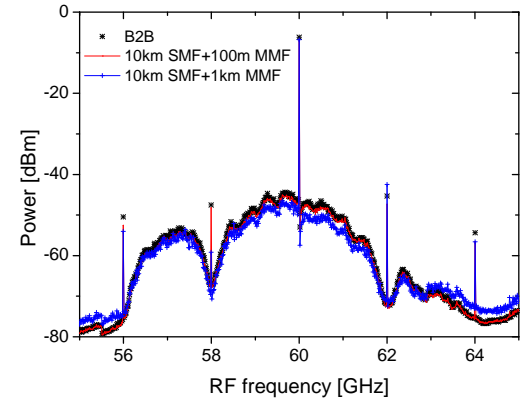

c)

Fig.3. a) Optical spectra at point A in Fig. 2, b) Phase noise of RF subcarriers and c) RF spectra of detected 60-GHz signals with 2-Gbps data at point B in Fig. 2 and $-3.0-\mathrm{dBm}$ received optical power.

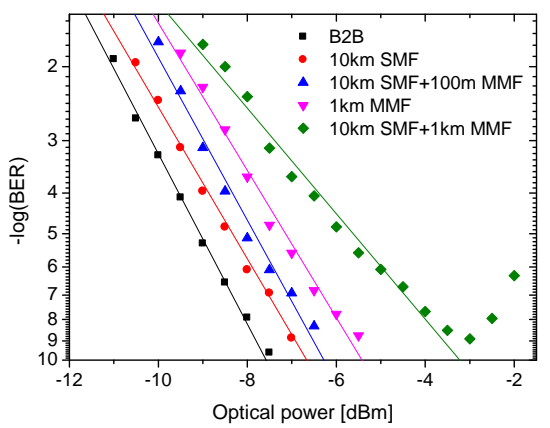

a)

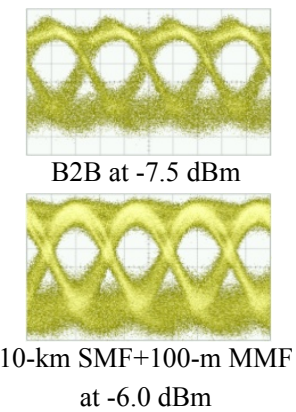

b)

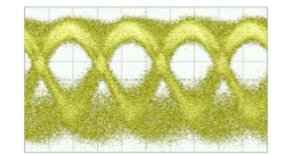

10-km SMF at $-6.5 \mathrm{dBm}$

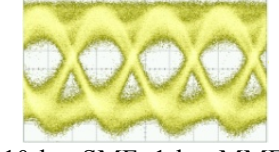

10-km SMF+1-km MMF

at $-4.0 \mathrm{dBm}$

Fig. 4. Measured performance of the system: a) BER, b) eye-diagrams (amplitude: $83.5 \mathrm{mV} / \mathrm{div}$ and time: $200 \mathrm{ps} / \mathrm{div}$ )

\section{Conclusion}

We have experimentally demonstrated a MMW-over-combined-SMF/MMF system with simple 60-GHz transceiver based on direct modulation and envelope detection. Error-free transmission was achieved for 2-Gbps data at $60-\mathrm{GHz}$ after 10-km SMF, 1-km MMF and 6.5-m wireless. The experiment proves the feasibility of using SMF in optical access networks in combination with the existing MMF in buildings for distribution of $60-\mathrm{GHz}$ signal direct from a $\mathrm{CO}$ to remote in-building antennas enabling a cost-effective solution for next generation gigabit in-building wireless access networks.

\section{Acknowledgements}

We would like to thank Thomas Jul from Rohde \& Schwarz Danmark A/S for allowing us to use the FSU 67-GHz spectrum analyzer for the experiment.

\section{References}

[1]. J. Yu et al, "Optical millimeter-wave generation or up-conversion using external modulators," IEEE PTL, 18, 265-267 (2006).

[2]. Y. Le Guennec et al, "New optical microwave up-conversion solution in radio-over-fiber networks for 60-GHz wireless applications," IEEE/OSA JLT, 24, 1277-1282 (2006).

[3]. D. Zibar et al, "16 Gb/s QPSK wireless-over-fibre link in 75-110 GHz band with photonic generation and coherent detection," ECOC2010, Th.9.B.6, 2010.

[4]. G. H. Smith et al, "A millimeter-wave full-duplex fiber-radio star-tree architecture incorporating WDM and SCM," IEEE PTL., 10, 16501652 (1998).

[5]. TC48 - High Rate Wireless Communications, Ecma International, http://www.ecma-international.org/ memento/TC32-TG20-M.htm.

[6]. WirelessHD Consortium, http://www.wirelesshd.org

[7]. IEEE 802.15 Working Group for WPAN http://www.ieee802.org/15/.

[8]. A. Flatman, "In-premises optical fiber installed base analysis to 2007," IEEE 802.3 10 GBE over FDDI Grade Fiber Study, 2004.

[9]. A. M. J. Koonen, L. M. Garcia, "Radio-Over-MMF Techniques_Part II: Microwave to Millimeter-Wave Systems," IEEE/OSA JLT, 26, 2396-2408 (2008).

[10]. B. A. Khawaja, M. J. Cryan, "Millimetre-wave radio-over-fibre data transmission over multimode fibre," MOTL, 53, 254-256 (2011).

[11]. A. Nkansah et al, "Multilevel Modulated Signal Transmission Over Serial Single-Mode and Multimode Fiber Links Using Vertical-Cavity Surface-Emitting Lasers for Millimeter-Wave Wireless Communications," IEEE T MICROW THEORY, 55, 1219-1228, (2007) 